\title{
Experiências e impactos do aprendizado em Homeopatia: relatos de médicos egressos do Curso de Especialização em Homeopatia da FMJ
}

\author{
PALAVRAS-CHAVE \\ - Homeopatia \\ - Terapias Cognitivas \\ - Humanizações da assistência \\ médica \\ - Educação em medicina \\ - Sistema Único de Saúde \\ - História oral
}

\section{KEY WORDS}

- Homeopathy

- Complementary Therapies

- Humanization of Assistance

- Medical education

- Single Health System

- Oral history

\author{
Experiences and Impacts of Learning \\ Homeopathy: reports by medical graduates of a \\ specialization course in homeopathy
}

Maristela Schiabel Adler ${ }^{\mathrm{I}}$

Dante Marcelo Claramonte Gallian ${ }^{\text {II }}$

\section{R E S U M O}

Nas últimas décadas, a insatisfação com o cuidado médico contribuiu para a valorização de formações médicas mais abrangentes e humanizadas e para a busca das medicinas complementares e alternativas (CAM) como opções de tratamento. Desde 2003, o Curso de Pós-Graduação em Homeopatia da Faculdade de Medicina de Jundiaí (PGH-FMJ) oferece o ensino da Homeopatia para médicos, integrando-a à medicina convencional. Este estudo analisa as experiências de aprendizado em Homeopatia dos egressos das duas primeiras turmas do Curso de Especialização em Homeopatia da PGH-FMJ, totalizando 14 participantes. A metodologia compreendeu questionários de múltipla escolha, entrevistas não estruturadas e a técnica de História Oral. Os resultados revelaram que os egressos adquiriram competências para o exercício da especialidade, com ganhos nas dimensões afetivas, intelectivas e de atitude, maior satisfação profissional e pessoal, e melhora na relação médico-paciente.

\section{A B S T R A C T}

In recent decades, dissatisfaction with medical care has led to greater appreciation of a more comprehensive and humanistic medical education and to the search for other treatment options among complementary and alternative medicines (CAM). Since 2003, the Jundiaí School of Medicine offers graduate education in homeopathy (PGH-FMJ) for medical doctors, combining homeopathy and conventional medicine. The aim of this paper was to analyze the learning experiences of graduate students from the two first classes at the PGH-FMJ, with a total of 14 participants. The methodology included multiple-choice questionnaires, non-structured interviews, and the oral history technique. The results showed that the learning of homeopathy at the Jundiaí School of Medicine developed specific professional skills and led to new humanistic, affective, intellectual, and attitudinal insights among participants, as well as to greater professional and personal satisfaction and an improved physician-patient relationship.

\footnotetext{
${ }^{I}$ Faculdade de Medicina de Jundiaí, Jundiaí, São Paulo, Brasil.

${ }^{I I}$ Universidade Federal de São Paulo, São Paulo, São Paulo, Brasil.
} 


\section{INTRODUÇÃO}

O desenvolvimento da pesquisa científica no século 20 trouxe importantes contribuições para os saberes da área da saúde. Porém, a complexidade dos novos conhecimentos cindiu o ensino generalista, fracionando-o e deslocando o foco de atenção para múltiplas especialidades. O organismo passou a ser compreendido, estudado e tratado por especificidade de órgãos e doenças. A mente foi retirada do corpo, adquirindo identidade de órgão à parte, "uma entidade mais espiritual que científica"1(p.48)

O ensino compartimentalizado passou a não exigir conhecimentos abrangentes sobre o ser humano, colaborando para uma prática médica setorial e técnica e afastando o médico do seu doente $^{2-4}$. O olhar atento da observação clínica individualizada e a sensibilidade na relação médico-paciente cederam espaço aos avanços da medicina tecnológica ${ }^{1,5-7}$.

Nas últimas décadas, a insatisfação com o cuidado médico fez com que pacientes buscassem alternativas de tratamento ${ }^{8-10}$. O ato médico e o próprio profissional tornaram-se alvo de críticas pela impessoalidade, compartimentalização e automatiza-

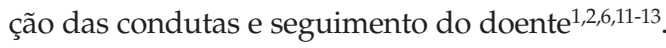

Hoje, em busca de uma formação médica integral, a Organização Mundial da Saúde (OMS) e órgãos responsáveis pelo ensino médico oficial preconizam uma formação humanizada que valorize o conhecimento geral do profissional e o pensamento crítico e reflexivo $^{14-18}$.

\section{O ENSINO DAS MEDICINAS COMPLEMENTARES E ALTERNATIVAS}

Na América do Norte, a partir de 2000, 41 escolas de Medicina introduziram em seus currículos e/ou iniciaram programas assistenciais, cujos princípios regentes são práticas de medicinas complementares e alternativas (Complementary and Alternative Medicine-CAM). O propósito foi enfatizar a relação médico-paciente e "integrar o melhor das Medicinas Complementares e Alternativas ao melhor da Medicina Convencional”8 (p.851), buscando proporcionar diferença qualitativa no cuidado à saú$\mathrm{de}^{19,20}$.

No Brasil, em 2003, teve início um curso de pós-graduação em Homeopatia na Faculdade de Medicina de Jundiaí (PGH-FMJ), com a finalidade de promover o ensino da Homeopatia hahnemanniana para médicos graduados, integrando-o à medicina convencional e ao Sistema Único de Saúde (SUS), como preconizado pelo Ciplan ${ }^{21}(1988)$ e pela Portaria 971 do Ministério da Saúde ${ }^{22}$ (2006).

A Homeopatia, parte integrante das CAM, teve sua origem no século 18. Samuel Hahnemann, seu criador, destacando a so- berania da clínica e da experimentação, desenvolveu um novo sistema terapêutico baseado na experiência racional e sistematizada, visando à prática de uma medicina integral, voltada para o doente como um todo e estruturada na individualidade de cada paciente ${ }^{23}$. Registrou seus principais avanços clínico-farmacêuticos em seis edições sucessivas de seu Organon da Arte de Cu$\operatorname{rar}^{24}$.

\section{OBJETIVO}

Esta pesquisa foi apresentada como dissertação de mestrado no programa de pós-graduação no Ensino em Ciências da Saúde da Escola Paulista de Medicina/Unifesp. Teve como objetivo analisar as experiências de aprendizado dos egressos do Curso de Especialização da Pós-Graduação em Homeopatia (lato sensu) da Faculdade de Medicina de Jundiaí (PGH-FMJ) e o impacto nas vidas profissionais desses ex-alunos, avaliando concepções de ordem afetiva, intelectiva e de atitude.

\section{OBJETO DE ESTUDO}

O Curso de Especialização da PGH-FMJ (http:/ / www.audesapere.com.br/) tem em seu conteúdo programático: História e Filosofia, Clínica Homeopática, Semiologia, Farmácia Homeopática, Matéria Médica Homeopática e Metodologia Científica. Com duração de dois anos, procura articular ensino e ambiente de trabalho ${ }^{25}$. A prática em ambulatórios vinculados ao SUS abrange $45 \%$ da carga horária total do curso, visando atendimento individualizado e humanizado. Busca também promover interação multiprofissional construtiva com outros profissionais da área da saúde, objetivando melhores cuidados ao doente ${ }^{26,27}$.

O principal foco do curso é o ensino do método clínico-terapêutico deixado por Hahnemann na sexta edição do Organon, denominado aqui Homeopatia hahnemanniana, cuja prática é caracterizada pelo uso das potências cinquenta-milesimais ${ }^{28}$. Recentemente, estas potências têm sido utilizadas também em estudos clínicos controlados, com resultados promissores no tratamento de doenças crônicas, não infecciosas ${ }^{29-32}$.

Participaram da pesquisa os médicos formados nas duas primeiras turmas da especialização em Homeopatia, totalizando 14 egressos.

\section{MÉTODOS}

A metodologia utilizada compreendeu questionários de múltipla escolha ${ }^{33}$ para respostas rápidas e objetivas, entrevistas não estruturadas $^{34}$, visando qualificar temas específicos, e a técnica de História Oral ${ }^{35-37}$. 
Os questionários e as perguntas das entrevistas não estruturadas abordaram três grandes eixos: o perfil do médico que ingressa no Curso de Especialização da PGH-FMJ; o trabalho médico homeopático dos egressos em serviços SUS e não SUS; e a estrutura do Curso de Especialização da PGH-FMJ.

No transcorrer da investigação, constatou-se que os questionários e entrevistas não respondiam satisfatoriamente aos questionamentos propostos, sendo preciso empregar outro instrumento metodológico para se obter uma abordagem específica e detalhada dos entrevistados. Hoje, os profissionais médicos, apesar de sofrerem frequentes críticas, são pouco convidados a expor suas opiniões. Um dos intentos desta pesquisa foi dar voz a este segmento que buscou o Curso de Especialização em Homeopatia da FMJ, para melhor compreensão de seus anseios, buscas, encontros e desencontros.

A técnica de História Oral mostrou-se uma ferramenta mais adequada para essa investigação, possibilitando uma compreensão ampla e minuciosa do papel da experiência do aprendizado, oferecido pelo curso, na vivência profissional e pessoal do egresso.

A História Oral fundamenta-se na apreensão de narrativas de experiências de um grupo, recolhendo testemunhos e promovendo análises de processos sociais do presente, com a finalidade de conhecer o meio proposto no estudo.

A elaboração da História Oral inicia-se com a definição de um projeto e de um grupo de pessoas a serem entrevistadas (comunidade de destino). Privilegia grupos sociais deslocados, parcelas marginalizadas e se vale de suas narrativas para propor uma história sobre determinada realidade, contrapondo-se ao silenciamento ou à visão majoritária e institucionalizada, assumida como aquela que devemos reconhecer como versão oficial.

A construção da História Oral prevê: estabelecimento da comunidade de destino, roteiro de perguntas, planejamento da condução das gravações (por meios eletrônicos), versão das falas gravadas para o escrito (transcrição-transcriação), conferência do texto com o depoente, autorização para o uso, arquivamento e, sempre que possível, publicação dos resultados, com o objetivo de dar retorno à comunidade de destino ${ }^{37}$.

As entrevistas devem ser realizadas em datas e horários predeterminados, com tempos equivalentes para todas. $\mathrm{O}$ assunto abordado deve ser exposto ao colaborador de forma clara e aberta, deixando-o à vontade para sua narrativa pessoal, evitando-se intervenções desnecessárias. Esta técnica de entrevista não diretiva não inclui o silêncio absoluto do entrevistador, mas uma posição interativa, em que o entrevistado dirige efetivamente o discurso, possibilitando a construção de uma fala de características ímpares, mais que o simples fornecimento de informações das entrevistas não estruturadas ${ }^{35}$. Sua realização não se reduz ao ato de entrevistar e gravar em aparelho eletrônico, mas transformar a entrevista em texto de valor documental. As emoções que envolvem a entrevista, captáveis em sua totalidade somente com a presença física, é algo que se procura transmitir nos textos escritos $^{36,37}$. É uma abordagem que possibilita uma visão abrangente ao valorizar, fundamentalmente, a trajetória de vida do sujeito, contrastando com as metodologias tradicionais, que apenas indicam, mas não revelam da mesma forma. Por meio desta técnica, os egressos puderam expor suas vivências singulares, auxiliando na compreensão desse específico processo de aprendizagem.

Para a obtenção de tais documentos, cada egresso foi entrevistado num segundo momento, com base num roteiro de perguntas que possibilitaram ampla exposição de opiniões e experiências vividas. (Fale sobre seus estudos em medicina e vivências profissionais, antes, durante e após o Curso de Especialização da PGH-FMJ. Como foi esta trajetória? Fale sobre sua prática hoje. Quais são seus planos para o futuro? Gostaria de acrescentar alguma coisa à sua narrativa?)

O tempo médio de cada entrevista foi de 50 minutos, e o material obtido nesta segunda entrevista foi trabalhado para se chegar a textos literários das Histórias Orais, como preconiza a técnica da História Oral ${ }^{37}$. Os entrevistados foram designados por pseudônimos (nomes de colaboradores do médico Samuel Hahnemann), para preservar suas identidades, evitando-se constrangimentos desnecessários.

Posteriormente, no processo de análise dos resultados, os dados obtidos por meio dos questionários e entrevistas não estruturadas, bem como as narrativas advindas da História Oral foram amplamente utilizados, permitindo uma rica visão das dimensões quantitativas e qualitativas.

\section{RESULTADOS}

Por meio do material obtido pôde-se constatar que todos os médicos que buscaram o Curso de Especialização da PGH-FMJ possuíam outras especialidades (Tabela 1), procurando pela medicina homeopática por motivos diversos (Tabela 2).

Ao ingressarem no curso, 79\% confessaram-se insatisfeitos com a profissão, dizendo-se desiludidos, frustrados e desmotivados com o exercício da medicina convencional. Mostraram-se descontentes com suas vidas profissionais, e os motivos se acham na Tabela 3. 
Tabela 1

Especialidades dos alunos ao ingressarem no Curso de Especialização da PGH-FMJ

\begin{tabular}{lcc}
\hline Especialidade & No de alunos & $\%$ \\
\hline Anestesiologia & 1 & 7 \\
Gastroenterologia & 1 & 7 \\
Ginecologia e Obstetrícia & 2 & 14 \\
Intensivista & 1 & 7 \\
Pediatria & 8 & 58 \\
Saúde da Família & 1 & 7 \\
\hline Total & 14 & 100 \\
\hline
\end{tabular}

Tabela 3

Motivos de descontentamento profissional dos entrevistados

\begin{tabular}{lcc}
\hline Motivos & No de alunos & $\%$ \\
\hline $\begin{array}{l}\text { Exposição a exaustivos regimes } \\
\text { de trabalho }\end{array}$ & 11 & 79 \\
$\begin{array}{l}\text { Compartimentalização do } \\
\text { tratamento médico }\end{array}$ & 9 & 64 \\
$\begin{array}{l}\text { Pensamento excessivamente } \\
\text { mercantilista da medicina atual }\end{array}$ & 6 & 43 \\
$\begin{array}{l}\text { Não reconhecimento da } \\
\text { abnegação profissional }\end{array}$ & 5 & 36 \\
$\begin{array}{l}\text { Perda do respeito dos pacientes } \\
\begin{array}{l}\text { Demasiada pressão das } \\
\text { indústrias farmacêuticas frente } \\
\text { às prescrições }\end{array}\end{array}$ & 3 & 21 \\
\hline
\end{tabular}

Eu não tinha tempo para nada e não conseguia resolver nada. Achava que não estava sendo a mãe que eu queria ser, a mulher que eu queria ser e a profissional que eu queria ser. Você não consegue resolver nada, tudo é limitado, tudo se limita. Você começa a encontrar limitação no trabalho, no tratamento do paciente, em você, limitação na pressão dos convênios médicos... (Melanie)
Tabela 2

Motivos de procura pela especialização em Homeopatia na FMJ

\begin{tabular}{lcc}
\hline Motivos & $\mathrm{N}^{0}$ de alunos & $\%$ \\
\hline $\begin{array}{l}\text { Falta de resolução da medicina } \\
\text { alopática que praticavam }\end{array}$ & 5 & 36 \\
Curiosidade & 4 & 29 \\
$\begin{array}{l}\text { Desejo de tratar o doente em sua } \\
\text { integralidade }\end{array}$ & 3 & 21 \\
$\begin{array}{l}\text { Procura pelo aprendizado da } \\
\text { Homeopatia hahnemanniana }\end{array}$ & 2 & 14 \\
\hline Total & 14 & 100 \\
\hline
\end{tabular}

Durante o aprendizado no Curso de Especialização, os egressos relataram necessidade de mudança dos conceitos aprendidos e enraizados na graduação. Citaram como aprendizado inédito a realização da anamnese homeopática, o aprender a ouvir o paciente, a abordagem sem preconceitos, o cuidado na observação do doente, o exame físico minucioso, a valorização de sinais e sintomas não valorizados pela medicina convencional, a forma de se entender o binômio saúde-doença, o enfoque de distúrbios pregressos do doente e o modo integral e individualizado de medicar e acompanhar o doente.

Aprendi a me preocupar mais com a qualidade da saúde e de vida da população em geral... Acho que hoje sou muito melhor que antes, porque eu tenho mais capacidade e ferramentas para obter um resultado melhor. (Luise)

Todos aprovaram o ensino prático da especialidade realizado integralmente no SUS e consideraram-se aptos para o início da vida na especialidade. Relataram a incorporação dos saberes homeopáticos às suas práticas alopáticas, com melhorias no modo de atendimento e entrevista do paciente, na forma de abordagem e na compreensão das patologias, no seguimento do caso, na disponibilidade, paciência e interesse pelo doente. A Homeopatia hahnemanniana, na opinião dos egressos, mostrou-se uma boa opção terapêutica por possibilitar o aumento da resolução clínica, principalmente em doentes crônicos, com redução da carga de medicamentos usada nesses casos. 
A Homeopatia me deu um instrumento que eu não tinha antes, para realmente eu me sentir cuidando do paciente e poder proporcionar um tratamento e uma cura adequados. (Wilhermine)

O embasamento científico do curso foi destacado como ponto positivo por $93 \%$ dos egressos.

A Tabela 4 mostra a avaliação feita pelos egressos de conhecimentos pertencentes ao currículo da graduação em Medicina ao iniciarem o curso.

Tabela 4

Temas considerados insuficientes durante a aprendizagem na graduação médica

\begin{tabular}{lcc}
\hline Tema & No de alunos & $\%$ \\
\hline Vacinação & 5 & 36 \\
$\begin{array}{l}\text { Uso de paliativos e } \\
\text { antibióticos }\end{array}$ & 7 & 50 \\
$\begin{array}{l}\text { Estrutura do texto } \\
\text { científico }\end{array}$ & 7 & 50 \\
$\begin{array}{l}\text { Conceitos de sintomas } \\
\begin{array}{l}\text { Conceito e compreensão } \\
\text { sobre pesquisas biomédicas }\end{array}\end{array}$ & 7 & 50 \\
Obstáculos à cura & 5 & 36 \\
\hline
\end{tabular}

As abordagens humanísticas do curso, como o estudo de Bioética, Filosofia, História da Homeopatia, História da Medicina e de medicinas não convencionais, foram aprovadas por todos os egressos, salientando-se que $64 \%$ criticaram a ausência deste aprendizado na graduação.

Em todas as narrativas, evidenciou-se aumento da satisfação profissional e pessoal com o exercício da nova especialidade. Ao iniciarem suas vidas como homeopatas, relataram-se médicos menos frustrados, mais felizes, gratificados, realizados e completos. Atribuíram o aumento da satisfação profissional aos bons resultados obtidos com o emprego do medicamento homeopático em seus pacientes, com maior grau de resolução nos tratamentos, trazendo-lhes a satisfação de praticar uma medicina com potencialidades não previamente concebidas por eles.

Meu contato com meu paciente é muito forte. Isto me faz hoje ter uma satisfação de estar no consultório, de ter uma consulta mais longa por um preço menor e de não levar a questão financeira como a única satisfação. (Eleonore)
Setenta e um por cento dos egressos mencionaram melhora na relação médico-paciente, atribuída à necessária abordagem diferenciada da Homeopatia hahnemanniana. Ao compararem suas práticas profissionais antes e depois do curso, revelaram aquisições positivas neste aprendizado, relatando-se mais humanizados.

A forma de atendimento humanizado proporcionou mudança na minha conduta de atendimento também na alopatia, antes muito técnica e com o direcionamento característico do alopata. (Friedrich)

Ainda do ponto de vista de relações humanas, todos mencionaram aquisição de novos predicados, como a compreensão perante outras formas reconhecidas de cuidado médico, incluindo as modalidades de CAM. Os colegas de curso foram elogiados por $64 \%$ dos entrevistados como auxiliares do aprendizado clínico e de relações profissionais. O acolhimento e as atitudes humanas dos orientadores foram considerados pontos construtivos do aprendizado profissional e de relações humanas por 79\%.

Quanto à minha evolução profissional e pessoal, a Homeopatia foi uma descoberta de um complemento formidável para abordar a pessoa, não só o doente. Isto transformou inclusive vários comportamentos meus, tanto profissionais como familiares e de relação interpessoal. (Gross)

Ao se dedicarem à prática homeopática, $64 \%$ puderam reduzir a carga de plantões e/ou os múltiplos empregos exigentes de produtividade, dispondo de maior tempo para o convívio familiar e para seus pacientes.

No momento da realização da pesquisa, constatou-se que todos os egressos se encontravam praticando a Homeopatia aprendida no curso.

\section{DISCUSSÃO}

Os resultados da pesquisa revelaram mudanças dos paradigmas previamente sedimentados pelos egressos.

Alterações na dimensão intelectiva foram evidenciadas na compreensão do adoecer, no emprego da medicação homeopática, no acompanhamento do doente e na valorização e tratamento de sintomas incompreensíveis à racionalidade da medicina convencional. Os estudantes, ao empregarem o método de anamnese homeopática, viram-se obrigados a abandonar a visão fracionada do órgão e buscar a totalidade do doente. A visão individual e integral 
do paciente foi mencionada por todos como fator de transformação pessoal. Na consulta homeopática, eles precisaram se dispor a ouvir todas as queixas do paciente sem preconceitos, a perguntar sobre sua vida e costumes. Isto se mostrou como novidade de árduo aprendizado. Ensinados a dirigir seus interrogatórios e avaliações durante a graduação, tiveram que reaprender a interrogar, a observar e examinar o paciente mais detidamente, pois tais subsídios são necessários ao tratamento integral e individualizado da Homeopatia hahnemanniana.

A experiência do contato com diferentes sofrimentos humanos por meio da consulta homeopática motivou a observação mais atenta dos egressos e estimulou a aproximação dos doentes, evidenciando-se ganhos nas dimensões volitivas e afetivas. A Homeopatia hahnemanniana valoriza aspectos físicos, mentais, emocionais e relacionais do paciente, de quaisquer patologias a serem tratadas. Eis a visão individual do todo ${ }^{24}$. Este reconhecimento do ser humano como unidade biopsicossocioespiritual é hoje apontado como lacuna do atual ensino médico ${ }^{38}$.

A prática homeopática no SUS, pela diversidade de doenças e volume de atendimento, contribuiu para maior capacidade na resolução de problemas e segurança clínica dos egressos. Os exemplos e atos dos responsáveis pelo ensino reforçaram o aprendizado humanístico, possibilitando sua sedimentação. Todos os entrevistados exerciam a Homeopatia hahnemanniana como especialidade médica no momento da pesquisa, divergindo de outros estudos que apontaram como dificuldade de recém-egressos de cursos de especialização em Homeopatia a não aquisição de habilidades específicas para o exercício da especialidade $^{39}$. As discussões clínicas estimularam atitudes reflexivas nos alunos, a busca de outros conhecimentos da própria medicina convencional e o aprimoramento das relações humanas. $\mathrm{Na}$ Homeopatia hahnemanniana, o exame do caso do doente, seu tratamento e seguimento, orientados por metodologia padronizada, possibilitaram o diálogo profissional, facilitando a troca de experiências $^{40}$.

Chamou a atenção dos pesquisadores o fato de temas curriculares da graduação serem considerados insuficientemente aprendidos pelos egressos, achado que requer novas investigações que fogem ao escopo deste estudo.

O aprendizado das humanidades (Bioética, Filosofia, História das Medicinas) trouxe novos saberes, estimulou o desenvolvimento de habilidades crítico-reflexivas e auxiliou na compreensão e análise de diferentes ciências, de suas aplicabilidades e possibilitou maiores questionamentos relativos à profissão que exerciam. Antes, eles se consideravam médicos de uma medicina de diagnóstico, técnica e fragmentada. Agora, relataram pra- ticar uma medicina integral e humanizada, com novas potencialidades terapêuticas, por eles não concebidas previamente.

Observou-se acréscimo de conhecimentos às competências dos egressos ao exercerem suas especialidades prévias ao curso, modificando abordagens e ampliando opções terapêuticas. Todos os entrevistados confirmaram o não abandono das especialidades já adquiridas, mas, sim, a aquisição de nova ferramenta que possibilita melhores cuidados aos pacientes em comparação à medicina convencional que antes realizavam.

Ganhos de ordem afetiva e atitudinal foram demonstrados no aumento da satisfação profissional com a prática da terapêutica adquirida e revelados na melhora da capacidade cuidadora, da tolerância, da dedicação e da responsabilidade individual e social dos egressos. Desiludidos e frustrados com a profissão ao ingressarem no curso, revelaram maior motivação e realização profissional ao praticarem e observarem os bons resultados obtidos com a Homeopatia hahnemanniana. Consideraram-se felizes e gratificados ao constatarem que, com a nova prática, puderam reduzir o número de empregos e plantões, melhorando o convívio familiar, a qualidade de suas vidas e a disponibilidade para com seus pacientes.

A abordagem individualizada reaproximou médicos e doentes, contribuindo significativamente para a melhora desta relação. Aprender a ser médico homeopata e ao mesmo tempo "conservador da saúde" ${ }^{\prime 24}$ (p.2) trouxe uma nova dimensão para os saberes afetivos destes egressos, mostrando que esta prática integral e individualizada auxilia a relação médico-paciente.

A satisfação profissional e a pessoal se entrelaçaram nas narrativas dos egressos ao se dizerem hoje mais felizes e médicos mais completos do que antes do curso. O aprendizado das ciências e das humanidades se complementou, auxiliando os egressos a desenvolver suas potencialidades.

Pôde-se observar nos egressos um acréscimo dos conhecimentos técnicos, clínicos, contextuais, relacionais, afetivo-morais e reflexivos - competências suficientes para o exercício da especialidade $^{15}$.

Como sugestão desta pesquisa, destaca-se a introdução de cursos informativos ou de formação homeopática, de metodologia semelhante, nas escolas de graduação médica. Sugere-se também a reintrodução dos estudos das humanidades nos currículos formais da graduação e de extensão universitária, na tentativa de se retornar a uma formação médica mais crítica e reflexiva, e contribuir para a humanização do médico. 


\section{CONCLUSÃO}

Nesta pesquisa, verificou-se a busca pelo Curso de Especialização da PGH-FMJ por médicos insatisfeitos com a profissão.

Todos os egressos consideraram suficientes os conhecimentos adquiridos no Curso de Especialização da PGH-FMJ para o início da vida profissional como homeopatas. No momento da pesquisa, estavam exercendo a nova especialidade médica, cujos saberes incorporaram também às práticas de suas especialidades prévias.

O Curso de Especialização da PGH-FMJ mostrou-se uma alternativa de ensino, apontando caminhos que se revelaram adequados ao aprendizado dessa especialidade.

Este estudo identificou mudanças afetivas, intelectivas e de atitudes, positivas nos egressos, que se apresentaram aos pesquisadores como respostas a um ensino coeso, de uma medicina cuja proposta é, desde a anamnese até o tratamento, a cura pela semelhança, priorizando a integralidade, a individualização do doente e um relacionamento humanizado.

\section{REFERÊNCIAS}

1. Lown, B. A arte perdida de curar. $3^{a}$ ed. brasileira. São Paulo: JSN Ed.;1996.

2. Gallian DMC. A (re)humanização da medicina. Psiquiatr Prat Med. 2000;33(2) 5-8.

3. Viesca C. Humanismo y medicina. Un Humanismo Médico para el Siglo XXI. In: Aréchiga H, (Org.). Ciência y Humanismo en Medicina. México: Editora Siglo XXI; 2003. p.71-89.

4. Aréchiga H. Ciência y Humanismo en Medicina. México: Editora Siglo XXI; 2003.

5. Coulehan JMD. Today's Professionalism: Engaging the Mind but Not the Heart. Acad Med. 2005;80(10)892-8.

6. Machado MH (Coord.). Os Médicos no Brasil: um retrato da realidade. Rio de Janeiro: Fiocruz; 1997.

7. Pereira Neto AF. Ser médico no Brasil, o presente no passado. Rio de Janeiro: Fiocruz; 2001.

8. Maizes V, Schneider C, Bell I, Weil A. Integrative medical education: development and implementation of a compreehensive curriculum at the University of Arizona. Acad Med. 2002; 77(9)851-60.

9. Salles SAC. As motivações dos médicos para a especialização em Homeopatia. Rev Bras Educ Med 2005;29(3)167-73.

10. Luz MT. A arte de curar versus a ciência das doenças. História Social da Homeopatia no Brasil. São Paulo: Dynamis; 1996.
11. Caprara A; Franco ALS. The patient-physician relationship: towards humanization of medical practice. Cad Saúde Pública. 1999;15(3)647-54.

12. Campos GWS. Educação médica, hospitais universitários e o Sistema Único de Saúde. Cad. Saúde Pública. 1999;15(1)187-94.

13. Macnaughton RJ, Evans HM. Medical humanities and medical informatics: an unlikely alliance? Is there a role for patients' voices in the modern case record? Med Humanit. 2004;30(2)57-8.

14. Organização Pan-Americana da Saúde. Organização Mundial da Saúde. Declaração de Alma-Ata. Conferência Internacional sobre cuidados primários de Saúde. [documento na internet]. URSS, 1978. [acesso em 11 ago. 2006]. Disponível

em http:/ / www.opas.org.br/coletiva/carta.cfm.

15. Epstein RM, Hundert EM. Defining and assessing professional competence. JAMA. 2002;287(9)226-35.

16. Batista NA, Batista SH. A docência em saúde: desafios e perspectivas. In: Batista NA, Batista $\mathrm{SH}$, (Org.). Docência em Saúde: temas e experiências. São Paulo: Ed. Senac; 2004. p.17-31.

17. Grossman E, Cardoso MHCA. As narrativas em medicina: contribuições à prática clínica e ao ensino médico. Rev Bras Educ Méd. 2006;30(1)6-14.

18. Ribeiro RJ. Apresentação do Organizador. In: Humanidades; um novo curso na USP. São Paulo: EDUSP; 2001. p.11-30.

19. Consortium of Academic Health Centers for Integrative Medicine.[on line]. [Acesso em 10 ago. 2008] Disponível em http://www.inconsortium.org/cahcim/members/home.html

20. Haramati A, Elder W, Heitkemper M, Warber S. Insights from Educational Initiatives in Complementary and Alternative Medicine. Acad Méd. 2007;82(10)919-20. 21.

Brasil. Ministério da Previdência e Assistência Social. Resolução Ciplan no4, de 8 de março de 1988. Fixar diretrizes sobre o atendimento médico Homeopático nos serviços públicos. Diário Oficial da União. Brasília, 11 mar. 1988;Seção 1, p. 3996-7.

22. Brasil. Ministério da Saúde. Portaria no 97, de 3 de maio de 2006. Aprova a Política Nacional de Práticas Integrativas e Complementares (PNPIC) no Sistema Único de Saúde. Diário Oficial da União. Brasília, 4 mai 2006; Seção 1, p.20. 
23. Luz MT. Medicina e racionalidades médicas: estudo comparativo da medicina ocidental contemporânea, homeopática, tradicional chinesa e ayurvédica. In: Canesqui AM, (Org.). Ciências Sociais e Saúde para o ensino médico. São Paulo: Hucitec; FAPESP; 2000. p.181-200.

24. Hahnemann CFS. Organon da arte de curar, 1842. Traduzido da $6^{\underline{a}}$ ed. alemã por David Castro, Rezende Filho e Kamil Curi. 2aed. brasileira. São Paulo: GEHSP "Benoit Mure"; 1995.

25. Brasil. Ministério da Educação e do Desporto. Parecer $n^{\circ}$ 908 de 2 de dezembro de 1998. Assunto: especialização em área profissional. [documento na internet]. Brasília;1998. [acesso em 02 set. 2008]. Disponível em http://portal.mec.gov.br/sesu/arquivos/pdf/Parecer908.pdf.

26. Nedrow AR, Heitkemper M, Frenkel M, Mann D, Wayne $\mathrm{P}$, Hughes E. Collaborations between Allopathic and Complementary and Alternative Medicine Health Professionals: Four Initiatives. Acad Med. 2007;82(10)962-6.

27. Galhardi WMP. A formação do médico homeopata na Faculdade de Medicina de Jundiaí: uma prática de ensino no SUS. Campinas; 2005. Mestrado [Dissertação] -Universidade de Campinas.

28. Adler UC, Cesar AT, Adler MS, Alves A, Garozzo EN, Galhardi WMP, et al. LM or Q-potencies: Review of Their Use Over a Fifteen-Year Period. Homeopathic Links. 2005;2(18)87-91.

29. Bell IR, Lewis DA, Brooks AJ, Schwartz GE, Lewis SF, Walsh BT, et al. Improved clinical status in fibromyalgia patients treated with individualized homeopathic remedies versus placebo. Rheumatology. 2004; 43(5): 577-82.

30. Frei H, Everts R, Ammon KV, Kaufmann F, Walther D, Hsu-Schmitz S, et al. Homeopathic treatment of children with attention deficit hyperactivity disorder: a randomised, double blind, placebo controlled crossover trial Euro J Pediatri..2005;164(12):758-67.

31. Adler UC, Paiva NM, Cesar AT, Adler MS, Molina A, Calil HM. Tratamento homeopático da depressão: relato de série de casos. Rev Psiquiatr Clínica (São Paulo). 2008;35(2):74-8.

32. Adler UC, Paiva NMP, Cesar AT, Adler MS, Molina A, Padula AE, Calil HM. Homeopathic individualized Q-poten- cies versus fluoxetine for moderate to severe depression: double-blind, randomized noninferiority trial.

33. Marconi MA, Lakatos EM. Técnicas de Pesquisa. $6^{a}$ ed. São Paulo: Atlas; 2006.

34. Lüdke M, André MED. Pesquisa em Educação: Abordagens Qualitativas. São Paulo: EPU; 1986.

35. Gallian DMC. Pedaços da Guerra: experiências com História Oral de vida de Tobarrenhos. São Paulo; 1992. Mestrado [Dissertação] - Universidade de São Paulo.

36. Meihy JCSB. Manual de História Oral. $5^{\mathrm{a}}$ ed. São Paulo: Edições Loyola; 2005.

37. Meihy JCSB, Holanda F. História Oral: como fazer, como pensar. São Paulo: Contexto; 2007.

38. Siqueira JE. a arte perdida de cuidar. Rev Bioet (Impr.). 2002;10(2)89-106.

39. Salles SAC. Perfil do Médico Homeopata. São Paulo; 2001. Mestrado [Dissertação] - Universidade de São Paulo.

40. Maia JA. Formação humanística no ensino superior em saúde: intencionalidade e acasos. In: Batista NA, Batista SH, Abdalla IG, (Orgs.). Ensino em Saúde: visitando conceitos e práticas. São Paulo: Arte e Ciência Editora; 2005. p.39-54.

\section{CONTRIBUIÇÃO DOS AUTORES}

O artigo resume Tese de Mestrado - defendido na UNIFESP, na qual fui a autora principal e o Prof. Dr. Dante M.C. Gallian, orientador.

\section{CONFLITO DE INTERESSES}

Declarou não haver

\section{ENDEREÇO PARA CORRESPONDÊNCIA}

Maristela Schiabel Adler

Pós-Graduação em Homeopatia - Faculdade de Medicina de Jundiaí
Av. Rouxinol, 1041 - cj.808
Moema - São Paulo
CEP.: 04516-902 SP
E-mail: madler@uol.com.br 